\title{
ArcheoSocial. L'archeologia riscrive il web: esperienze, strategie e buone pratiche, A cura di Antonia Falcone \& Astrid D'Eredità, DiElle Editore 2018
}

http://www.dielleditore.com/archeosocial.html

\section{Reviewed by Paola Di Giuseppantonio Di Franco}

University of Essex

La sintesi di questo volume è sapientemente offerta nel titolo: AcheoSocial è una contrazione di 'Archeologia Sociale', un'archeologia che sappia parlare di sé, attraverso gli strumenti della comunicazione contemporanea, primi fra tutti social media e blog. Il volume è unico nel suo genere e si propone come strumento di conoscenza e comprensione delle principali piattaforme di divulgazione archeologica online e dei linguaggi comunicativi ad esse associati, fungendo da manuale per principianti e al tempo stesso fornendo approfondimenti legati a esempi virtuosi o buone pratiche di comunicazione online in ambito archeologico in Italia.

Perché è importante che gli esperti raccontino l'archeologia in Italia oggi? Per smantellare i cliché legati alla disciplina, certo, quelli che ci definiscono avventurieri, scopritori di tesori e dinosauri, o persino alchimisti in possesso di verità occulte legate ai maggiori misteri dell'archeologia nel mondo. Ma anche per dare impulso e lustro alla professione di specialista dei Beni Culturali, il cui ruolo di valorizzazione e tutela del patrimonio culturale Italiano viene oggi spesso minato da campagne politiche e mediatiche svilenti e svalutative. Infine, per promuovere l'archeologia dal basso, con la convinzione che l'alto interesse di una comunità per il proprio patrimonio rappresenti l'arma più efficace di protezione, promozione e valorizzazione del patrimonio stesso.

Raccontare l'archeologia attraverso blog e social media significa poter raggiungere velocemente un pubblico vasto, a patto che lo si faccia utilizzando linguaggi adatti e strategie digitali efficaci. ArcheoSocial rappresenta un utile strumento per comprendere quali siano le migliori strategie di comunicazione online e offre spunti importanti per la creazione di piani editoriali mirati alla promozione. Il volume, come spiegato in prefazione, rappresenta il punto d'approdo scritto di una serie di workshop sul tema organizzati dalle curatrici a TourismA-Salone Archeologia Culturale, fiera annuale della comunicazione archeologica, organizzata a Firenze a partire dal 2015.

Il volume è curato da Antonia Falcone e Astrid D'Eredità, due archeologhe esperte di comunicazione digitale, che hanno scelto di raccogliere una selezione di saggi scritti da sole donne, per contrastare una pratica diffusa che vede troppo spesso l'uomo in archeologia come principale soggetto narrante, nonostante la percentuale di archeologhe in Italia superi di gran lunga quella degli uomini. Uno studio del 2014 
(DISCO) citato da A. Falcone nel capitolo introduttivo Dal blog ai social: una strategia digitale integrata per la narrazione archeologica (p. 11, nota 3), ha infatti rivelato che in Italia le donne rappresentavano il $70.79 \%$ degli archeologi professionisti, contro il 29. 21\% di uomini.

Il volume si compone di una prefazione, scritta da Piero Pruneti, direttore della rivista di informazione archeologica Archeologia Viva (http://www.archeologiaviva.it/), e nove capitoli.

Il già citato capitolo di Antonia Falcone elucida le scelte contenutistiche e editoriali sottese al volume e introduce la cassetta degli attrezzi del social manager, tra cui, il principale resta la trowel; non può esistere social manager in archeologia che non abbia usato la trowel almeno una volta, ovvero che non si sia professionalizzato sul campo.

I capitoli dal 2 al 5 descrivono, nello specifico, le principali piattaforme digitali utilizzate per la divulgazione dei contenuti archeologici, fornendo dettagli e consigli su linguaggi e strategie di comunicazione associati a ciascuna di queste piattaforme. Il messaggio principale di questa parte del libro è che l'uso delle piattaforme proposte dovrebbe essere il più possibile integrato e basato su un preciso piano editoriale. Quest'ultimo prevede: una strategia che includa degli obiettivi comunicativi di partenza; una riflessione sull'audience a cui indirizzare i contenuti; la selezione delle piattaforme di condivisione da utilizzare, ai cui linguaggi specifici cui andranno adattati i contenuti stabiliti durante i piani editoriali giornalieri, settimanali e mensili; la valutazione del successo dei post e l'eventuale cambiamento di strategia derivante dall'analisi dei dati di gradimento (i.e., analytics and insights, pp. 22, 32-33). Molto utili sono gli esempi offerti all'interno dei capitoli, che illustrano la gamma di strategie a disposizione per l'aumento di visibilità e livello di interazione delle comunità online con i propri post; uno fra tutti, forse ovvio ai nativi digitali, ma non a professionisti che, come me, si sono da poco avvicinati al web come forma di comunicazione, legare i propri Tweet ai trending topic (TT) del giorno, come spiega Marta Coccoluto nel capitolo Quando la comunicazione mette le ali: archeologia e musei su Twitter (p. 57).

I capitoli 6, 7 e 8 del volume illustrano le buone pratiche della comunicazione archeologica online, attraverso la descrizione dettagliata di casi studio virtuosi, come Roma 3 Scava la pagina Facebook legata agli scavi in atto presso l'Anfiteatro Flavio e il Foro della Pace e diretti da Riccardo Santangeli Valenzani e la Sovrintendenza di Roma presentata nel capitolo Dietro le quinte di 'Roma 3 scava': esperienze di comunicazione social per l'archeologia di Giulia Facchin, Brigitte Budani e Rachele Buonomo. Il capitolo Open Salapia: il progetto di archeologia pubblica dello scavo di Salapia (Trinitapoli) di Giovanna Baldasarre racconta di Open Salapia, un progetto di archeologia pubblica legato al sito di Salapia, per il quale l'account Twitter e la pagina Facebook rappresentano un ponte tra la comunicazione online e quella off line, sul sito. Il caso di Aquinum presentato nel capitolo Incrementare la conoscenza di un sito archeologico: dall'online all'offline. Il caso di Aquinum scritto da Giovina Caldarola mostra un progetto di integrazione di tutte le maggiori piattaforme di social media per una comunicazione efficace del sito e offre un altro sapiente esempio di come la come la comunicazione online possa servire da ponte per 
attività di archeologia pubblica sul sito. Infine, il capitolo scritto da Marianna Marcucci, Elisa Bonacini e Cettina Santagati \#InvasioniDigitali: co-creazione di valore culturale attraverso la partecipazione attiva dei pubblici alla narrazione archeologica illustra il portale \#InvasioniDigitali che dà voce alle comunità di persone che supportano il patrimonio culturale invadendo musei e siti e documentando l'esperienza via web. Quest'ultimo caso studio presentato nel volume è un progetto di ricerca, oltre che di comunicazione. Nato per valorizzare il patrimonio Italiano, vanta oggi una community molto più ampia di "invasori", a livello soprattutto europeo. Oltre a fornire un esempio virtuoso di comunicazione e valorizzazione del patrimonio dal basso, \#InvasioniDigitali rappresenta soprattutto un modello per coloro che come me, si interessano di progetti di archeologia pubblica che prevedano la co-creazione di contenuti digitali sul patrimonio. \#InvasioniDigitali può essere definito a tutti gli effetti un progetto di archeologia pubblica digitale. Una delle invasioni descritte ha visto coinvolti due gruppi di studenti delle università di Catania e Palermo, che hanno co-creato e diffuso repliche tridimensionali digitali di artefatti antichi; le risposte degli studenti a un questionario post-esperienza sembrano suggerire come la co-produzione di contenuti archeologici destinati ai social media favorisca una connessione più intima con il patrimonio e l'acquisizione di nuove conoscenze durante il processo di produzione del contenuto stesso.

Nel capitolo conclusivo al volume, Astrid D'Eredità e Giovanna Falcone riflettono sul futuro di blog e social media in ambito archeologico, proponendo approcci lungimiranti "per dare una patina di professionismo ad un'attività spesso oggetto di improvvisazione" (p. 191).

Il tono narrativo di tutti gli autori di Archeosocial è tendenzialmente positivo ed enfatizza il potenziale della comunicazione via web, lasciando al lettore la facoltà di coglierne i limiti, che vengono accennati in modo indiretto e trasversale lungo tutto il volume. I limiti sono principalmente associati ad un uso poco consapevole, poco etico e poco professionale degli strumenti di comunicazione proposti e ad un uso iperspecialistico del linguaggio o, viceversa, all'uso di un linguaggio sensazionalistico già ampiamente diffuso in ambito giornalistico e televisivo quando si parla di archeologia. Considerato l'intento educativo del volume l'analisi di casi studio poco etici e meno virtuosi avrebbe senza dubbio offerto spunti di riflessione importanti soprattutto a un'utenza studentesca e questa mancanza è, a mio avviso, uno dei limiti del volume.

Visto il numero di tecnicismi e termini specialistici presenti nel volume, molto utili sarebbero stati anche un glossario e un indice analitico in appendice.

Il volume offre buoni spunti bibliografici per chi voglia approfondire argomenti specifici. A tali riferimenti credo sia opportuno aggiungere una recente raccolta di saggi sul tema dei blog in archeologia: il numero speciale di Internet Archaeology Journal a cura di Colleen Morgan e Judith Winters.

La lettura di questo volume edito ha rappresentato per me un importante momento di riflessione e autocritica sul modo in cui io ho finora utilizzato i social media per la 
promozione dei miei progetti e la comunicazione di contenuti digitali. Il libro mi ha messo di fronte ai limiti della mia linea comunicativa online, che è sicuramente deficitaria di un chiaro piano editoriale e una pianificazione dei contenuti che mantenga costante l'attenzione dei miei utenti ai miei canali di comunicazione.

Ho trovato la lettura stimolante e mi sono ritrovata spesso a prendere appunti e gridare "eureka!", ogniqualvolta il libro offrisse spunti creativi ai miei problemi di comunicazione online.

Sono fiduciosa che questo lavoro possa offrire stimoli futuri per la produzione di simili pubblicazioni sull'argomento, che focalizzino anche l'attenzione sugli effetti della comunicazione social dell'archeologia Italiana sulla protezione e sulla valorizzazione del nostro patrimonio. ArcheoSocial è decisamente un volume di riferimento per tutti coloro che lavorano nel campo della promozione, protezione e valorizzazione del patrimonio culturale in Italia. Il linguaggio del volume è semplice, diretto e scorrevole, tanto da rendere questo un utile strumento non solo per gli esperti nel settore, ma anche per gli studenti che si avvicinano per la prima volta all'utilizzo dei social media ai fini della comunicazione archeologica.

\section{References}

DISCO Discovering Archaeologists of Europe 2014 a cura della Confederazione Italiana Archeologi, https://www.discovering-archaeologists.eu

Morgan C., \& J. Winters, 2015. Introduction: Critical Blogging in Archaeology. Internet Archaeology 39. https://doi.org/10.11141/ia.39.11 